\title{
Hormônios sexuais, moralidades de gênero e contracepção de emergência no Brasil
}

Elaine Reis Brandão(a)

Brandão ER. Sex hormones, gender moralities and emergency contraception in Brazil. Interface (Botucatu). 2018; 22(66):769-76.

The aim of the present study is to discuss different social judgments about the growing use of sex hormones, a practice that is increasingly common in Brazil and worldwide. There has been a rapid expansion of clinical indications for the use of sex hormones in different circumstances, such as aging, beauty purposes and improvement of physical and sexual performance. These technological innovations are disseminated by the media enthusiastically. Paradoxically, there is some reserve regarding the use of emergency contraception by young women. Despite the popularization of scientific knowledge of sex hormones as a tool to improve some physical human aspects, the diffusion and use of emergency contraception, which was approved twenty years ago with precise clinical indications, remain marginal and restricted, in a country where abortion is still unavailable to women. The study hypothesis considers a gender perspective that subjugates the exercise of women's sexuality to specific prevailing standards.

Keywords: Gonadal steroid hormones. Contraception. Sexuality. Gender. Contraception methods.
Propõe-se discutir juízos sociais distintos sobre o crescente uso de hormônios sexuais, para fins contraceptivos ou não, prática cada vez mais abrangente no contexto internacional e no Brasil. Há uma expansão vertiginosa de indicações clínicas para uso de hormônios sexuais em diferentes circunstâncias da vida (como envelhecimento, embelezamento, melhoria de performance sexual e física), sendo tais inovações tecnológicas propagadas com entusiasmo pela mídia. Paradoxalmente, convive-se com certa reserva ao uso da contracepção de emergência pelas jovens mulheres. A despeito da popularização do conhecimento científico sobre hormônios sexuais como fontes de aprimoramento humano, a difusão e uso da contracepção de emergência, aprovados há vinte anos no Brasil, com indicações clínicas precisas, continuam marginais e sofrendo restrições, em um país no qual o aborto segue interditado às mulheres. A hipótese considera uma perspectiva de gênero que subjuga o exercício da sexualidade feminina a determinados padrões morais vigentes.

Palavras-chave: Hormônios sexuais. Anticoncepção. Sexualidade. Gênero. Métodos contraceptivos. (a) Departamento de Medicina Preventiva, Instituto de Estudos em Saúde Coletiva, Universidade Federal do Rio de Janeiro (UFRJ). Avenida Horácio Macedo, s/ $n^{\circ}$, Praça da Prefeitura Universitária da UFRJ, Ilha do Fundão, Cidade Universitária. Rio de Janeiro, RJ, Brasil. 21941-598. brandao@iesc.ufrj.br 


\section{Introdução}

Nas últimas décadas, a difusão, o consumo e a utilização de hormônios sexuais conheceram uma expansão sem precedentes, muito além do tratamento de transtornos menstruais e de suas finalidades contraceptivas ${ }^{1-3}$. Hormônios sexuais foram concebidos pela endocrinologia no início do século $X X$ como mensageiros químicos da feminilidade e da masculinidade. Essa compreensão do corpo humano regido pelos hormônios buscava responder às origens das diferenças sexuais localizando-as no âmbito da fisiologia ${ }^{3}$. No entanto, o debate público sobre tal expansão no campo da Saúde Coletiva encontra-se ainda muito incipiente.

Esse fenômeno somente pode ser compreendido no âmbito de questões culturais e socioeconômicas mais amplas que contextualizam o crescente uso de medicamentos nas sociedades de carizes técnico-científicas contemporâneas. Há um sólido debate acadêmico internacional e nacional sobre a expansão do processo de medicalização nas sociedades ocidentais modernas, a partir de trabalhos seminais de Foucaultt, ${ }^{4,5}$, Ilich $^{6}$, Zola ${ }^{7}$ e Conrad'. Segundo o último autor, define-se medicalização como "um processo pelo qual problemas não médicos passam a ser definidos e tratados como problemas médicos, frequentemente em termos de doenças ou transtornos"1. Dentre as inúmeras peculiaridades que configuram tal processo na atualidade, destaca-se a ampliação da difusão e consumo de medicamentos e tecnologias médicas, mediados pela relação direta entre os laboratórios médicos e farmacêuticos e os consumidores (potenciais pacientes). A crescente aproximação entre público usuário e tecnologias médicas ou farmacológicas tem sido promovida pelo marketing farmacêutico ou biomédico pela web, fomentando a disseminação de produtos, artefatos e medicamentos a potenciais consumidores ${ }^{8}$. Tal debate aponta ainda para o uso de medicamentos não propriamente para tratamento e prevenção de doenças, mas principalmente para o aprimoramento humano (enhancement), ou seja, para a melhoria e aperfeiçoamento das capacidades físicas, psíquicas e de vitalidade dos sujeitos sãos ${ }^{8-12}$. As perspectivas teóricas em debate enfatizam aspectos particulares dessa grande engrenagem social que nos captura, como biomedicalização ${ }^{(b)}$ ou farmaceuticalização ${ }^{(c)}$ da sociedade ${ }^{13}$, mas sobressai sempre a tendência de uso de medicamentos no governo de $\mathrm{si}^{1^{14}}$.

O objetivo do texto é discutir distintos gradientes de aceitação social, valorização e restrições ao uso dos hormônios sexuais, conforme gênero e posição social. O exemplo da contracepção de emergência funciona como um objeto "bom para pensar" a respeito do modo como as diferenças de gênero e de moralidades sexuais incidem nas representações e práticas de saúde.

\section{Hormônios sexuais: fetiches de uma nova era?}

Três aspectos exemplificam o argumento de ampliação significativa da utilização dos hormônios sexuais na vida cotidiana dos indivíduos, sejam estes cisgêneros ou transgêneros, de diferentes idades e classes sociais, para fins de aprimoramento biomédico ou contraceptivo. Em geral, os medicamentos são utilizados ou prescritos off label, ou seja, fora da indicação constante na bula, ou protocolo, para uso ainda não aprovado(d)16,17. O primeiro diz respeito à crescente prescrição e utilização da pílula anticoncepcional oral, de uso diário, as chamadas pílulas de última geração, que possuem outros fins que não a prevenção da gravidez. Sua consagração como "droga de estilo de vida"18,19 a torna recomendável para uma série de circunstâncias, com indicações clínicas dermatológicas e estéticas, para melhoria do humor, etc.

\footnotetext{
(b) "Biomedicalização

descreve a crescente complexidade, multissituacionalidade e multidimensionalidade dos processos de medicalização, ampliados e reconstituídos por meio de novas formas sociais de biomedicina altamente tecnocientífica. [...] Cinco principais processos interativos engendram a biomedicalização e são por ela também produzidos: 1 - a reconstituição econômico-política do vasto setor de biomedicina; 2- o foco na saúde em si e a elaboração dos padrões de risco e de vigilância biomédicos; 3- a natureza cada vez mais tecnológica e científica da biomedicina; 4transformações no modo como os conhecimentos biomédicos são produzidos, distribuídos e consumidos; e no gerenciamento de informações médicas; e 5- transformações nos corpos para incluir novas propriedades e a produção de novas identidades tecnocientíficas individuais e coletivas. Assinalamos com o prefixo "bio", em "biomedicalização", as transformações tanto humanas quanto não humanas, tornadas possíveis por inovações tecnocientíficas como biologia molecular, biotecnologias, genomização, medicina de transplantes e novas tecnologias médicas. Ou seja, a medicalização está se intensificando, mas sendo consolidada sob novas, complexas e tecnocientíficas formas" 15 (tradução minha).
} 
(c) "Farmaceuticalização denota a tradução ou transformação de condições, recursos e capacidades humanas em oportunidades de intervenção farmacêutica. Esses processos podem se expandir muito além dos domínios estritamente médicos ou medicalizados ${ }^{1}$ para abranger outros usos não médicos para fins de melhoria do estilo de vida, aumento de capacidades ou aprimoramento humano (entre pessoas 'saudáveis')." 14 (tradução minha)

(d) Para uma discussão mais detalhada sobre usos off label de medicamentos, Stafford ${ }^{20}$ e Radley et al. ${ }^{21}$
O segundo alude à crescente recomendação, prescrição e utilização massiva de hormônios sexuais para finalidades de aprimoramento humano, ou seja, melhoria de sua performance vital, seja física ou sexual|16,17, em atividades de fitness, de potencialização dos corpos em transformação, melhoria do humor, bem-estar, prevenção do envelhecimento, entre outros exemplos. A medicalização da menopausa, com a introdução da terapia de reposição hormonal nas mulheres, na década de 1980, e da Deficiência Androgênica do Envelhecimento Masculino (DAEM), ou andropausa ${ }^{22}$, fenômeno mais recente, são exemplos concretos de expansão do uso dos hormônios sexuais na vida cotidiana.

Por fim, o terceiro aspecto aborda a indicação de métodos contraceptivos reversíveis de longa duração (Long-Acting Reversible Contraception - Larc) para adolescentes de 15 a 19 anos usuárias do Sistema Único de Saúde (SUS). As recentes consultas públicas disponibilizadas pela Comissão Nacional de Incorporação de Tecnologias no SUS (Conitec), n 35 e no $36^{23}$, em 2015, sobre uma eventual introdução no SUS dos métodos contraceptivos reversíveis de longa duração, solicitadas pela Federação Brasileira das Associações de Ginecologia e Obstetrícia (Febrasgo) ao Ministério da Saúde (MS), atualizam o debate a respeito da inclusão de novas alternativas contraceptivas às mulheres usuárias da rede pública de serviços de saúde.

Trata-se da oferta de dois dispositivos contraceptivos às mulheres entre $15 \mathrm{e}$ 19 anos: o implante subdérmico liberador de etonogestrel, com duração de três anos, e o sistema intrauterino liberador de levonorgestrel, com duração de cinco anos. A Federação defende a introdução dessa modalidade de contraceptivos na rede pública de saúde, com indicações para uso em "populações especiais", como adolescentes, usuárias de drogas ilícitas e mulheres convivendo com vírus da imunodeficiência humana ${ }^{24}$. Embora em 2016 o Ministério da Saúde tenha se posicionado contrário à solicitação feita pela Febrasgo, a discussão sobre as indicações clínicas e vantagens dos Larc cresce a cada dia no país, tendo em vista as elevadas taxas de gravidez imprevista, a vulnerabilidade de determinados estratos sociais e a alegação de que tais métodos "não dependem da disciplina da mulher" 25 .

Decerto, existem muitas controvérsias científicas, sociais e éticas que emolduram essa expansão, incluindo desde sua propaganda viral pela web por parte dos laboratórios farmacêuticos até a crescente automedicação pelos sujeitos, sem o necessário acompanhamento clínico e a valorização da autonomia individual como um bem em si mesmo, desassociada de um contexto estrutural que a possibilite. Não menos importante, há também uma cultura, um certo ethos de que o corpo passou a ser algo plástico, moldável, flexível para ser transformado, o que abre portas infinitas para sua remodelação pela via dos hormônios sexuais e das intervenções ou cirurgias estéticas. ${ }^{26}$

\section{Dois pesos, duas medidas?}

No entanto, se por um lado convive-se com certa euforia na utilização indiscriminada de hormônios na vida cotidiana, por outro, paradoxalmente, há no país certa reserva ao uso da contracepção de emergência pelas jovens mulheres, em geral concebido de modo negativo, como uma "bomba hormonal" 27,28. A despeito de uma popularização do conhecimento científico sobre os hormônios sexuais como fontes de aprimoramento humano, bem como potencialidade anticonceptiva, a difusão e o uso da contracepção de emergência, aprovado há vinte anos no Brasil, com indicações clínicas precisas - como falha conhecida ou presumida do método em uso de rotina, não uso ou uso inadequado do método 
contraceptivo e violência sexual - continuam marginais e sofrendo restrições, em um país no qual o aborto segue interditado às mulheres ${ }^{29,30}$.

Coexistem, então, distintos juízos sociais e morais entre as várias modalidades de utilização de hormônios, ressaltados e recomendados, por um lado, e condenados ou aceitos com reservas, por outro. Passados vinte anos da introdução da contracepção de emergência no Brasil (inicialmente na fórmula Yuspe e, em seguida, com o levonorgestrel), por que permanecem tantas restrições ao seu uso e disseminação nos serviços públicos de saúde? Por que tais reservas recaem neste contraceptivo específico (pós-coito), e não no conjunto de dispositivos tecnológicos e medicamentos hormonais em franca ascensão na sociedade? Por que os "riscos" à saúde feminina, os "perigos" que a ingestão de hormônios pode representar ao corpo das mulheres, tendem a ser levantados em relação à contracepção de emergência em particular, e não em sentido amplo, em relação ao conjunto dos métodos contraceptivos hormonais? A alegação de uma "dose maior ou excessiva" de hormônios nela contida, condensada no imaginário social pela expressão "bomba hormonal", não se sustenta pelas evidências científicas disponíveis ${ }^{31-33}$.

Em geral, os riscos à saúde não são mencionados quando os hormônios sexuais são utilizados para preservar ou reforçar um "dever ser" da vitalidade (física e sexual), da juventude e da beleza em homens e mulheres, muitas vezes sem acompanhamento clínico devido ${ }^{34,17}$. O exemplo da medicalização do corpo dos homens, a partir dos 35-40 anos, na chamada Daem ${ }^{1,22}$ (ou andropausa), não desperta tanta celeuma quanto o uso da contracepção de emergência entre mulheres. $\mathrm{O}$ uso da testosterona pelos homens (ou mulheres) não é contestado da mesma forma que a contracepção de emergência. Decerto, o reforço à virilidade masculina e ao bom desempenho sexual são valores caros à manutenção de uma identidade de gênero masculina hegemônica, ao passo que a introdução dos métodos contraceptivos reversíveis de longa duração aparece no espaço público com a justificativa de corrigir a indisciplina das mulheres, relapsas com o uso da contracepção no cotidiano ${ }^{35}$. E nenhuma discussão pública é levantada sobre a responsabilidade masculina com a contracepção. O debate sobre contracepção revela sempre uma face perversa da desigualdade de gênero.

O que subjaz a uma condenação moral mais enfática do uso da contracepção de emergência por mulheres adolescentes e jovens, em especial, de segmentos excluídos da sociedade brasileira? Por que os riscos à saúde não são levantados em relação, por exemplo, aos implantes subcutâneos hormonais, em geral utilizados por celebridades, modelos e mulheres de alto poder aquisitivo ${ }^{17}$

Algumas hipóteses são apontadas para problematizar a desigualdade entre as apreciações públicas por parte de profissionais de saúde, usuários, gestores e prescritores sobre diferentes modos de utilização dos hormônios sexuais, assinalando especificidades que envolvem o estatuto da contracepção de emergência ${ }^{36}$. Tais reflexões remetem ao que Carrara ${ }^{37}$ designa como "nova geografia do mal e do perigo sexual", quando o autor discute as políticas sexuais no Brasil e seus peculiares estilos de regulação moral. Ao analisar as transformações no dispositivo de sexualidade ${ }^{4}$, constituído por uma anatomopolítica dos corpos e uma biopolítica das populações, que transita de uma sexualidade vinculada à reprodução para a sexualidade como prazer e forma de subjetivação, consolidada na noção de "direitos sexuais", Carrara ressalta como as dimensões de responsabilização e de controle de si ganham relevância. E são exatamente essas noções que estão no cerne do debate sobre a contracepção pós-coito ou sobre os contraceptivos de longa duração.

No caso da contracepção de emergência, o exercício da sexualidade feminina, principalmente na adolescência, é colocado em xeque, sob suspeição, excetuando-se as circunstâncias de violência sexual, ainda que não sejam raras as suspeitas sobre o comportamento sexual feminino em atos de estupro no Brasil. A sexualidade adolescente é sempre pensada como fora de controle e imprevidente, redundando em gravidezes e abortos. Recrimina-se a "negligência" ou "displicência" das mulheres que não se preveniram antecipadamente à relação sexual, utilizando um método contraceptivo para se evitar a gravidez. Condena-se a busca pelo contraceptivo de emergência nos serviços públicos de saúde e nas drogarias do país, não se valorizando tal iniciativa como um cuidado em saúde por parte das mulheres. Em geral, a contracepção pós-coito é considerada como uma preocupação "tardia" por parte das mulheres que não se preveniram antecipadamente à relação sexual ${ }^{27,28}$. Em um contexto no qual vige a ilegalidade do aborto ${ }^{29,30}$, a morbimortalidade decorrente de práticas abortivas inseguras 
e uma epidemia de Zika vírus em curso $^{38}$, a centralidade desse método contraceptivo deveria ser maior. São inúmeras as dificuldades de acesso ao contraceptivo de emergência em tempo oportuno no Sistema Único de Saúde (até 120 horas após a relação sexual) ${ }^{39}$. A educação em sexualidade, que poderia problematizar o exercício sexual na adolescência e na juventude e as questões de gênero a ele vinculadas, continua não sendo um direito assegurado, nem amparado pelo Estado brasileiro. As últimas discussões sobre a exclusão das categorias de "gênero" e "orientação sexual" do Plano Nacional de Educação (PNE) e da Base Nacional Comum Curricular, recém-aprovada pelo Ministério da Educação, comprovam as dificuldades do debate sobre sexualidade e gênero no âmbito da educação pública.

Por estar situada em um continum de três tecnologias reprodutivas hormonais, ou seja, entre a pílula anticoncepcional oral, de uso diário, e os medicamentos utilizados no aborto terapêutico (misoprostol e mifepristona), o estatuto da contracepção de emergência inclui a dúvida no centro de sua gênese ${ }^{36}$. A controvérsia sobre sua configuração dúbia a constitui. Sua posição intermediária entre ambos os dispositivos hormonais a faz deslizar para os dois extremos, conforme os argumentos religiosos, científicos, técnicos ou políticos em jogo. O fato de ser (erroneamente) considerada por uns com efeitos abortivos e, por outros, um medicamento hormonal como outro qualquer, instaura uma tensão que não se dispersa. O estatuto moral desse objeto carrega em si tal controvérsia, dificultando sobremaneira sua aceitação social mais ampla ${ }^{40}$.

No caso dos contraceptivos de longa duração, recomendados especialmente às mulheres adolescentes e jovens pobres, a indisciplina feminina se soma à chamada "imaturidade" juvenil, corroborando os estereótipos de gênero, geração e classe social que recaem nas adolescentes de segmentos excluídos da sociedade brasileira. Supõe-se ser impossível que estas mesmas adolescentes possam aprender a ser responsáveis e a exercitarem o autocontrole, daí uma solução de longo prazo, que independa de sua ingerência.

Por fim, os constrangimentos de gênero que imperam em uma sociedade hierarquicamente estruturada como a brasileira, em favor do masculino, definem normas de condutas sociais e sexuais distintas para homens e mulheres, refletindo em censura moral quando o sexo é praticado pelas mulheres, fora do casamento e sem o devido planejamento prévio. Tal apreensão acaba por isentar a participação dos homens na responsabilidade contraceptiva no âmbito de relacionamentos afetivosexuais ou de relações sexuais eventuais. Embora o uso do preservativo masculino em todas as relações sexuais não seja uma prática consensual no Brasil, o adjetivo de "indisciplina" costuma ser atribuído às mulheres, e nunca aos parceiros homens.

\section{Considerações finais}

A falta de legitimidade e institucionalidade que cerca a contracepção de emergência, embora regulamentada pelo Estado brasileiro no fim da década de 1990, faz pensar sobre os limites ou as tensões de gênero na definição de uma sexualidade feminina socialmente aceitável. A discriminação das usuárias como "meninas perdidas" ou "sem-vergonha" 41 aponta para a regulação social dos corpos femininos, em especial, jovens e pobres, nos quais a reprodução é temida. E, principalmente, expressa as dificuldades que as mulheres enfrentam em assegurar que elas próprias possam, ao seu modo, controlar sua fecundidade, dispondo de qualquer método que lhe convier, conforme sua fase de vida, condições de parceria (ocasional ou estável) e situação socioeconômica, sem necessariamente precisarem ser tuteladas por especialistas em razão de sua "indisciplina". O aprendizado da "responsabilidade" e do "controle de si" é gradual e perpassa as práticas educativas das instituições de saúde e de educação. Portanto, os conteúdos relativos à sexualidade e ao gênero não podem ser suprimidos nas políticas públicas de educação - tema também em pauta. A proposta de inclusão dos métodos contraceptivos de longa duração para as adolescentes não pode prescindir de considerá-las como sujeitos em processo de autonomização capazes de aprender a se cuidar.

O debate sobre as hierarquias de gênero na sociedade brasileira e o quanto tais hierarquias em favor do masculino geram sofrimento, adoecimento e discriminação às mulheres não pode estar 
ausente da agenda da Saúde Coletiva no que tange à discussão sobre o planejamento reprodutivo na contemporaneidade ${ }^{42}$. A defesa intransigente do acesso universal ao aborto seguro e do direito a uma assistência integral e de qualidade para que mulheres em geral possam ser respeitadas e amparadas em suas escolhas contraceptivas, a consideração dos distintos momentos do ciclo de vida, a disponibilidade dos insumos e o acompanhamento clínico devido são dimensões que precisam ser pautadas com urgência.

Referências

1. Conrad P. The medicalization of society: on the transformation of human conditions into treatable disorders. Baltimore: John Hopkins University Press; 2007.

2. Harding J. Sex and control: the hormonal body. Body Soc. 1996; 2(1):99-111.

3. Oudshoorn N. Beyond the natural body: an archeology of sex hormones. London: Routledge; 1994.

4. Foucault M. História da sexualidade I: a vontade de saber. 13a ed. Rio de Janeiro: Graal; 1999.

5. Foucault M. Historia de la medicalización. Educ Méd Salud. 1977; 11(1):3-25.

6. Illich I. A expropriação da saúde: nêmesis da medicina. Rio de Janeiro: Nova Fronteira; 1975.

7. Zola IK. Medicine as an institution of social control. Soc Rev. 1972; 20(4):487-504.

8. Dumit J. Drugs for life. How pharmaceutical companies define our health. Durham: Duke University Press; 2012.

9. Camargo Jr KR. Medicalização, farmacologização e imperialismo sanitário. Cad Saude Publica. 2013; 29(5):844-6.

10. Martin E. The pharmaceutical person. BioSocieties. 2006; 1(3):273-87.

11. Rohden F. Vida saudável versus vida aprimorada: tecnologias biomédicas, processos de subjetivação e aprimoramento. Horiz Antropol. 2017; 23(47):29-60.

12. Zorzanelli R, Ortega F, Bezerra Jr B. Um panorama sobre as variações em torno do conceito de medicalização entre 1950-2010. Cienc Saude Colet. 2014; 19(6):1859-68.

13. Williams SJ, Martin P, Gabe J. The pharmaceuticalisation of society? A framework for analysis. Sociol Health III. 2011; 33(5):710-25.

14. Rose N. A política da própria vida: biomedicina, poder e subjetividade no século XXI. São Paulo: Paulus; 2013.

15. Clarke AE, Shim JK, Mamo L, Fosket JR, Fishman JR. Biomedicalization: technoscientific transformations of health, illness, and U.S. biomedicine. Am Soc Rev. 2003; 68(2):161-94.

16. Hoberman J. Testosterone dreams: rejuvenation, aphrodisia, doping. Berkeley: University of California Press; 2005.

17. Manica D, Nucci M. Sob a pele: implantes subcutâneos, hormônios e gênero. Horiz Antropol. 2017; 23(47):93-129.

18. Nucci M. Seria a pílula anticoncepcional uma droga de estilo de vida? Ensaio sobre o atual processo de medicalização da sexualidade. Sex Salud Soc. 2012; 10:124-39. 
19. Watkins ES. How the pill became a lifestyle drug. The pharmaceutical industry and birth control in the United States since 1960. Am J Public Health. 2012; 102(8):1462-72.

20. Stafford RS. Regulating off-label drug use. Rethinking the role of the FDA. New Engl J Med. 2008; 358(14):1427-9.

21. Radley DC, Finkelstein SN, Stafford RS. Off-label prescribing among office-based physicians. Arch Intern Med. 2006; 166(9):1021-6.

22. Rohden F. "O homem é mesmo a sua testosterona": promoção da andropausa e representações sobre sexualidade e envelhecimento no cenário brasileiro. Horiz Antropol. 2011; 17(35):161-96.

23. Consultas públicas n. ${ }^{\circ 35}$ e n. ${ }^{\circ 36}$. Disponíveis em: http://conitec.gov.br/index.php/ consultas-publicas-2015-encerradas

24. Penna IAA, Brito MB. A importância da contracepção de longo prazo reversível. Femina. 2015; 43(1):1-6.

25. Posicionamento da Comissão Nacional de Anticoncepção da FEBRASGO sobre a decisão do MS. Disponível em: http://www.febrasgo.org.br/site/?p=12305.

26. Edmonds A, Sanabria E. Medical borderlands: engineering the body with plastic surgery and hormonal therapies in Brazil. Anthropol Med. 2014; 21(2):202-16.

27. Brandão ER, Cabral CS, Ventura M, Paiva SP, Bastos LL, Oliveira NVBV, et al. "Bomba hormonal": os riscos da contracepção de emergência na perspectiva dos balconistas de farmácias no Rio de Janeiro, Brasil. Cad Saude Publica. 2016; 32(9):e00136615.

28. Brandão ER, Cabral CS, Ventura M, Paiva SP, Bastos LL, Oliveira NVBV, et al. Os perigos subsumidos na contracepção de emergência: moralidades e saberes em jogo. Horiz Antropol. 2017; 23(47):131-61.

29. Diniz D, Medeiros M, Madeiro A. Pesquisa nacional de aborto 2016. Cienc Saude Colet. 2017; 22(2):653-60.

30. Heilborn ML, Cabral CS, Brandão ER, Faro L, Cordeiro F, Azize RL. Itinerários abortivos em contextos de clandestinidade na cidade do Rio de Janeiro - Brasil. Cienc Saude Colet. 2012; 17(7):1699-708.

31. Cleland K, Raymond EG, Westley E, Trussell J. Emergency contraception review: evidence-based recommendations for clinicians. Clin Obstet Gynecol. 2014; 57(4):741-50.

32. Trussell J. Emergency contraception: hopes and realities. In: Foster A, Wynn LL, editores. Emergency contraception: the story of a global reproductive health technology. New York: Palgrave Macmillan; 2012. p. 19-35.

33. Westley E, Glasier A. Emergency contraception: dispelling the myths and misperceptions. Bull World Health Organ. 2010; 88(4):243-4.

34. Silva AC. "Limites" corporais e risco à saúde na musculação: etnografia comparativa entre duas academias de ginástica cariocas [tese]. Rio de Janeiro (RJ): Universidade Federal do Rio de Janeiro; 2014.

35. Brandão ER. Métodos contraceptivos reversíveis de longa duração no Sistema Único de Saúde: o debate sobre a (in)disciplina da mulher. Cienc Saude Colet [Internet]. 2017 [citado 26 Jul 2017]. Disponível em: http://www.cienciaesaudecoletiva.com.br/artigos/ metodos-contraceptivos-reversiveis-de-longa-duracao-no-sistema-unico-de-saude-odebate-sobre-a-indisciplina-da-mulher/16251.

36. Wynn LL, Trussell J. The social life of emergency contraception in the United States: disciplining pharmaceutical use, disciplining sexuality, and constructing zygotic bodies. Med Anthropol Q. 2006; 20(3):297-320.

37. Carrara S. Moralidades, racionalidades e políticas sexuais no Brasil contemporâneo. Mana. 2015; 21(2):323-45. 
38. Baum P, Fiastro A, Kunselman S, Vega C, Ricardo C, Galli B, et al. Garantindo uma resposta do setor de saúde com foco nos direitos das mulheres afetadas pelo vírus Zika. Cad Saúde Pública. 2016; 32(5):e00064416.

39. Souza RA, Brandao ER. Marcos normativos da anticoncepção de emergência e as dificuldades de sua institucionalização nos serviços públicos de saúde. Physis. 2009; 19(4):1067-86.

40. Isambert FA. Une sociologie de l'avortement est-elle possible? Rev Fr Soc. 1982; 23(3):359-81.

41. Paiva SP, Brandao ER. Silêncio e vergonha: contracepção de emergência em drogaria do Rio de Janeiro. Rev Estud Fem. 2017; 25(2):617-36.

42. Brandão ER, Cabral CS. Da gravidez imprevista à contracepção: aportes para um debate. Cad Saude Publica. 2017; 33(2):e00211216.

Brandão ER. Hormonas sexuales, moralidades de género y contracepción de emergencia en Brasil. Interface (Botucatu). 2018; 22(66):769-76.

Se propone discutir juicios sociales distintos sobre le creciente uso de hormonas sexuales, para fines contraceptivos o no, práctica cada vez más incluyente en el contexto internacional y en Brasil. Hay una expansión vertiginosa de indicaciones clínicas para la utilización de hormonas sexuales en diferentes circunstancias de la vida (tales como envejecimiento, embellecimiento, mejora del desempeño sexual y físico), siendo tales innovaciones tecnológicas propagadas con entusiasmo por los medios. Paradojalmente, se convive con cierta reserva al uso de la contracepción de emergencia por parte de las mujeres jóvenes. A pesar de popularización del conocimiento científico sobre hormonas sexuales como fuentes de perfeccionamiento humano, la difusión y el uso de la contracepción de emergencia, aprobados desde hace veinte años en Brasil, con indicaciones clínicas precisas, continúan marginalizados y sufriendo restricciones en un país en el cual el aborto sigue siendo prohibido para las mujeres. La hipótesis considera una perspectiva de género que subyuga el ejercicio de la sexualidad femenina a determinados estándares morales vigentes.

Palabras clave: Hormonas sexuales. Contracepción. Sexualidad. Género. Métodos contraceptivos.

Submetido em 05/05/17. Aprovado em 03/10/17. 